\title{
Estrategia de educación popular para promover la participación comunitaria en la prevención del dengue en Cuba
}

\author{
Lizet Sánchez, ${ }^{1}$ Dennis Pérez, ${ }^{1}$ Lázara Alfonso, ${ }^{1}$ Marta Castro, ${ }^{1}$ \\ Luis Manuel Sánchez, ${ }^{2}$ Patrick Van der Stuyft ${ }^{3}$ y Gustavo Kourí ${ }^{1}$
}

Forma de citar Sánchez L, Pérez D, Alfonso L, Castro M, Sánchez LM, Van der Stuyft P, et al. Estrategia de educación
popular para promover la participación comunitaria en la prevención del dengue en Cuba. Rev Panam popular para promover la particip
Salud Publica. 2008;24(1):61-9.

RESUMEN Objetivos. Documentar el proceso y analizar los resultados de la aplicación de una estrategia dirigida a aumentar la participación comunitaria en la lucha contra el mosquito vector del dengue.

Métodos. Entre mayo de 2002 y mayo de 2004 se aplicó una intervención para transformar la acción social contra el dengue en tres circunscripciones del municipio Playa, Ciudad de La Habana, Cuba. Se conformó un grupo de aprendizaje y grupos de trabajo comunitario (GTC) en las localidades. Se siguió un modelo de educación popular que conjugaba la puesta en práctica de las acciones previstas con sesiones de reflexión y aprendizaje a partir de los resultados y la reformulación del trabajo para las etapas subsiguientes. Se desarrollaron diagnósticos comunitarios, acciones preventivas, de comunicación, vigilancia y evaluación. Los cambios en la participación se identificaron mediante la técnica de análisis de contenido de documentos y entrevistas a informantes clave.

Resultados. El trabajo comunitario avanzó de acuerdo con las capacidades y los intereses de cada comunidad con enfoques de trabajo diferentes: de comunidad saludable, de riesgo ambiental y de riesgo entomológico. Se constataron cambios positivos en el concepto de participación según las cinco dimensiones evaluadas: liderazgo, identificación de necesidades, organización, gestión y movilización de recursos. Al final de dos años de intervención, el índice de depósitos con larvas o pupas de Aedes aegypti por 100 viviendas inspeccionadas se redujo en $79 \%$ y no se detectaron casos de dengue en ninguna de las circunscripciones.

Conclusiones. La estrategia empleada permitió reducir el nivel de infestación con el mosquito vector mediante el aumento de la participación de la comunidad en la toma de decisiones, el fortalecimiento de las competencias de los equipos médicos y de los GTC para liderar procesos participativos en sus comunidades y la elevación de la confianza de los miembros de la comunidad en sus posibilidades de llevar a cabo transformaciones con recursos propios y apoyo intersectorial.

Palabras clave Educación de la población, participación comunitaria, dengue, control vectorial, Aedes, Cuba.

1 Instituto de Medicina Tropical Pedro Kourí, Ciudad de La Habana, Cuba. La correspondencia se debe dirigir a Lizet Sánchez, Subdirección de Epidemiología, Instituto de Medicina Tropical Pedro
Kourí, Autopista Novia del Mediodía km 61/2, Apartado postal Marianao 13, Ciudad de La Habana, Cuba. Correo electrónico: lsanchez@ipk. sld.cu
2 Área de Salud 26 de Julio, Municipio Playa, Ciudad de La Habana, Cuba.

3 Unidad de Epidemiología, Instituto de Medicina Tropical, Amberes, Bélgica. 
El dengue constituye un problema de salud creciente en el mundo, especialmente en las Américas (1). En muchos países de la Región circulan diferentes serotipos virales causantes de esta enfermedad, incluso al mismo tiempo. Por otra parte, su principal vector, el mosquito Aedes aegypti, se encuentra ampliamente distribuido en el continente. Esto ha ocasionado que la enfermedad sea endémica en la mayoría de los países y que en muchos de ellos haya casos de dengue hemorrágico, la forma clínica más grave de la enfermedad (2).

Desde la década de 1990 se reconoce la importancia de involucrar a los miembros de las comunidades para lograr programas sostenibles de control, desde las etapas de identificación de las necesidades y las prioridades hasta el planeamiento y la ejecución de las acciones (3). Sin embargo, la mayoría de las estrategias diseñadas hasta el momento se han concentrado en elevar los conocimientos de la población sobre el dengue y su agente transmisor, pero no han logrado aumentar sustancialmente la participación de la comunidad en la eliminación de los sitios de cría de $A$. aegypti (4). Se ha argumentado que los cambios conductuales se logran a largo plazo y que requieren de estrategias de comunicación y movilización social que promuevan y refuercen conductas aceptadas por los miembros de las comunidades y que a su vez sean efectivas desde el punto de vista entomológico (5).

La guía de comunicación para el impacto conductual (COMBI), promovida por la Organización Mundial de la Salud y la Organización Panamericana de la Salud, propone los pasos a seguir para lograr cambios conductuales específicos relacionados con la eliminación de los principales sitios de cría del vector (6). La nueva generación de programas de prevención y control del dengue en las Américas aboga por un enfoque ecosistémico más integral, que se ha fundamentado en cuatro principios básicos: la voluntad política de los gobiernos, la coordinación intersectorial, la participación activa de la comunidad y el fortalecimiento de la legislación nacional (7).
Surge con esto, en los inicios del siglo XXI, un nuevo enfoque que se basa en la descentralización de la toma de decisiones y los recursos, y que da mayor importancia a la negociación de los comportamientos y a los cambios sociales (8).

Por otra parte, la educación popular - como concepto político-pedagógico nacido del pensamiento de Paulo Freire en la década de 1960 y enriquecido por la práctica latinoamericana actual- propone diversas formas de acción, a partir de un pensamiento crítico y creador que promueve a las personas como sujetos de los procesos sociales (9-11). Son pocas las experiencias de control de enfermedades que se basan en este concepto, sin embargo, las existentes se distinguen por su carácter motivador, su enfoque holístico e integrador de la realidad, su énfasis en el trabajo de grupos y el desarrollo de redes de apoyo, todo para alcanzar una acción transformadora en los miembros de la comunidad y lograr un mayor compromiso social. La educación se concibe no como un simple proceso de transmisión/adquisición de conocimientos, sino como una educación crítica, cuestionadora y transformadora de la realidad (12). Se privilegia el diálogo y la comunicación horizontal en el proceso de aprendizaje, así como la reinvención, la reformulación y el enriquecimiento permanente de las prácticas preventivas y de promoción de salud en las comunidades.

Cuba ha sido uno de los pocos países que ha logrado que el dengue no se establezca de forma endémica. En esto han incidido: 1) el Programa Nacional de Erradicación de A. aegypti, instaurado en 1981, que ha mantenido índices de infestación bajos en comparación con otros países de la Región; 2) el Programa Nacional de Control Sanitario Internacional, dirigido a detectar e impedir la introducción de nuevos focos de infección con este virus en el país; 3) las actividades de prevención y control desplegadas por la red de atención primaria de salud (APS); y 4) el enfoque intersectorial y multidisciplinario adoptado durante las campañas de control y eliminación de las epidemias (13). A pesar de los éxitos obteni- dos, aún quedan zonas con niveles de infestación con $A$. aegypti relativamente altos que han propiciado la aparición de brotes de dengue, principalmente en las provincias de Santiago de Cuba y Ciudad de La Habana $(14,15)$.

Este hecho ha llevado a las autoridades de salud a desarrollar en diversas localidades investigaciones piloto dirigidas a probar estrategias alternativas que permitan lograr una mayor coordinación intersectorial y una participación comunitaria más eficaz. En el presente trabajo se documenta el proceso y se analizan los resultados de la aplicación de una estrategia basada en el concepto de educación popular dirigida a aumentar la participación comunitaria en la lucha contra el mosquito vector del dengue en un área de salud de Ciudad de La Habana, Cuba.

\section{MATERIALES Y MÉTODOS}

\section{Área de estudio y antecedentes}

El estudio se llevó a cabo en las circunscripciones $^{4}$ 20, 51 y 40 del área de salud correspondiente a la policlínica 26 de Julio, del municipio Playa, Ciudad de La Habana, Cuba. En esta área de salud se ha mantenido la presencia de $A$. aegypti desde 1992 y ha habido casos de dengue durante todas las epidemias que han afectado a esta provincia. La estrategia descrita en el presente trabajo constituye la segunda fase de un proyecto piloto iniciado en 1999. La primera fase se centró en el fortalecimiento de alianzas y de la coordinación intersectorial mediante la capacitación de los miembros del consejo de salud. ${ }^{5}$ Los métodos y resultados de esa primera fase, que antecedió al presente trabajo, se publicaron con anterioridad (16).

Después de la capacitación recibida, el grupo intersectorial desarrolló una estrategia de comunicación y movili-

\footnotetext{
4 Las circunscripciones son la menor unidad políticoadministrativa en Cuba; agrupan aproximadamente entre 1000 y 1500 personas. Varias circunscripciones constituyen un consejo popular, compuesto por alrededor de $15000-25000$ personas.

5 Los consejos de salud son grupos intersectoriales que coordinan las acciones sanitarias en un consejo popular.
} 
zación social para el control del mosquito transmisor en todo el consejo popular, sin distinguir las diferencias existentes en el interior del mismo. Los métodos de control promovidos consistieron en la eliminación de recipientes no útiles de las viviendas y sus alrededores, el tapado de los tanques de agua y la limpieza de terrenos baldíos y áreas comunes. La evaluación realizada mostró un aumento en los conocimientos y mejoras en las prácticas de los pobladores con respecto al control de $A$. aegypti. Sin embargo, a pesar de que se enfocó la participación como un proceso mediante el cual las personas de la comunidad se involucran en la toma de decisiones, el planeamiento, la aplicación y la evaluación, con la estrategia aplicada solamente se logró elevar la participación de la población en las acciones y solo los miembros del consejo de salud intervinieron en la toma de decisiones, el planeamiento y la evaluación. A partir de la experiencia obtenida se decidió concebir una segunda fase y rediseñar la estrategia tomando en cuenta la participación activa de los pobladores y la heterogeneidad de las comunidades que integran el consejo de salud participante.

\section{Descripción de la estrategia}

Esta segunda fase del proyecto piloto se desarrolló entre mayo de 2002 y mayo de 2004. En la figura 1 se presenta el modelo conceptual de la estrategia seguida para elevar la participación comunitaria.

En este modelo se conjuga la puesta en práctica de las acciones previstas con sesiones de intercambio y aprendizaje a partir de la reflexión y el análisis crítico de las experiencias. Esto permitió reformular el trabajo de las etapas subsiguientes y elevar la conciencia sobre la problemática y las competencias de los participantes en la conducción de procesos participativos con vistas a solucionar los problemas existentes y elevar la calidad de vida de los pobladores. En esta segunda fase se distinguen tres componentes fundamentales: la organización para la participación, la capacitación y el trabajo comunitario.
Organización para la participación. En cada circunscripción se constituyó un grupo de trabajo comunitario (GTC) que coordinó las acciones a ese nivel. Esos grupos se encargaron de coordinar las actividades en las circunscripciones y de establecer vínculos con los miembros del consejo de salud. Cada GTC estuvo integrado por 8-10 líderes, entre ellos el delegado del Poder Popular de la circunscripción que es la máxima autoridad de gobierno en la circunscripción-, los médicos y los enfermeros o enfermeras de la familia, ${ }^{6}$ los representantes de las organizaciones sociales en la comunidad y otros líderes no formales. Para la ejecución de las actividades, los GTC se apoyaron en la red de líderes de las organizaciones sociales comunitarias existentes en cada cuadra (activistas de higienización, brigadistas sanitarios y otros líderes institucionales y no institucionales).

Adicionalmente, en el área de salud se constituyó un grupo de aprendizaje formado por los coordinadores de los GTC. Inicialmente, este equipo estuvo integrado por los médicos de la familia del área, pero posteriormente se incorporaron otros líderes naturales identificados por los mismos GTC. Estos equipos se reunieron trimestralmente para intercambiar y analizar las experiencias y elaborar propuestas para la reformulación del trabajo de los GTC.

Capacitación. La capacitación se concibió como un proceso continuo de aprendizaje que se inició con un taller para los coordinadores de los GTC. Este taller fue diseñado y coordinado por especialistas del Instituto de Medicina Tropical Pedro Kourí (IPK), de La Habana, y tuvo como objetivo preparar al personal de salud para promover la participación comunitaria en la prevención del dengue. Abarcó temas relacionados con el trabajo de grupo y la participación comunitaria e intersec-

\footnotetext{
En Cuba la atención primaria de salud está organizada a partir de médicos especialistas en medicina general integral que trabajan junto con un enfermero o enfermera directamente en las comunidades y atienden aproximadamente a 120 familias. Además de sus tareas asistenciales, realizan labores de prevención de salud.
}

torial en la identificación y la solución de los problemas ambientales relacionados con la proliferación del vector, la vigilancia de los casos de dengue y el diagnóstico participativo. A partir de este taller se planificaron encuentros trimestrales de capacitación, intercambio de experiencias y evaluación. Estos encuentros alternaron con la introducción en la práctica de los aspectos aprendidos y en ellos se profundizó en temas específicos de acuerdo con las necesidades de los participantes. Los temas abordados fueron, entre otros: la formación de GTC, el trabajo de grupo, el trabajo comunitario, la planeación estratégica, la comunicación popular, el diseño de actividades educativas y de estrategias de comunicación, la evaluación participativa y los cambios de comportamientos necesarios para el control de los principales criaderos de $A$. aegypti en la zona. Los encuentros se diseñaron siguiendo la lógica pedagógica de la educación popular: se parte de la práctica, se profundiza en la teoría y se regresa a la práctica transformada. Esta lógica conduce a los participantes a una apropiación consciente de sus prácticas y favorece los procesos de abstracción que les permiten comprenderlas de una manera diferente, para luego proyectarse en acciones transformadoras.

Trabajo comunitario. El trabajo comunitario se intercaló con la capacitación y permitió introducir lo aprendido en la práctica. Contó con cinco etapas: la identificación de los problemas, la planeación estratégica, el diseño y aplicación de una estrategia local de comunicación y movilización social, la vigilancia comunitaria del dengue y la evaluación participativa.

Identificación de los problemas. Los GTC discutieron los temas a abordar en el diagnóstico inicial, entre ellos, la identificación de los riesgos, los comportamientos asociados con los principales sitios de cría del vector y los problemas relacionados con la participación comunitaria. Con la ayuda de los especialistas del IPK se elaboraron instrumentos de recolección de información y un esbozo del plan de análi- 


\section{FIGURA 1. Modelo conceptual de la estrategia de educación popular empleada para aumentar la participación comunitaria en la prevención del dengue}

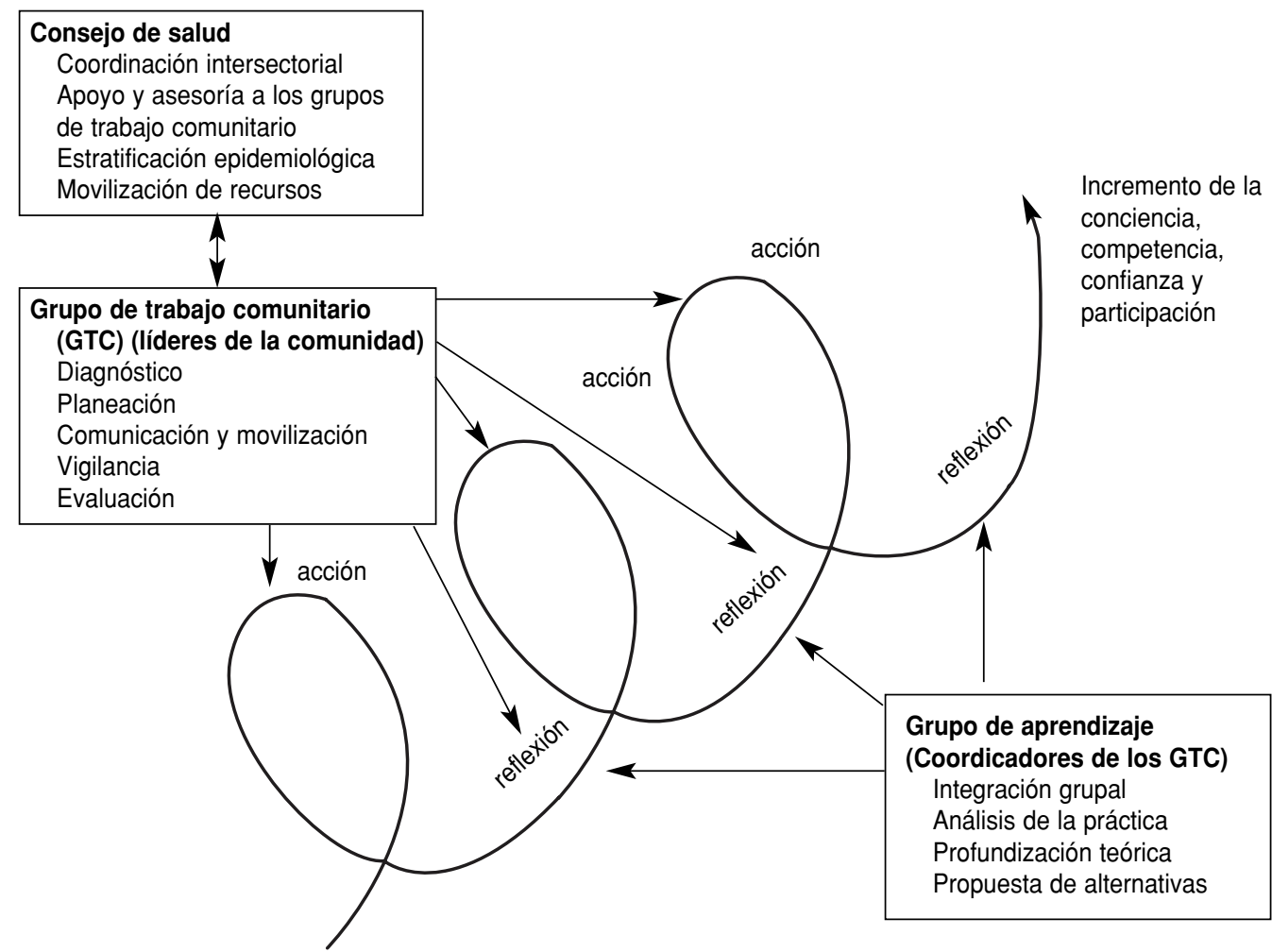

Fuente: Adaptado de Shrestha (17).

sis. En una reunión comunitaria se informó a la población de las actividades a realizar y se definió el cronograma. Como parte del diagnóstico se realizaron entrevistas de grupo, una encuesta a la población y visitas de observación a las viviendas. Una vez recolectada y procesada la información, se organizó un grupo de discusión con líderes de la comunidad para analizar los resultados y establecer las prioridades.

Planeación estratégica. A partir de los resultados del diagnóstico, los GTC elaboraron planes de acción para cada circunscripción. En estos se indicaban las actividades a realizar, el tiempo de ejecución, los responsables y los recursos necesarios. El grupo informó al consejo de salud sobre las decisiones tomadas en la circunscripción y solicitó recursos y apoyo para la realización de las actividades. A través del consejo de salud se gestionó la solución de los problemas que requerían de la intervención y de recursos de sec- tores e instituciones externos a la circunscripción. Un grupo de miembros de la comunidad formados como promotores de salud negociaron con los residentes en el área la solución de los problemas encontrados en las viviendas y promovieron comportamientos específicos, tales como el cepillado de los tanques y la autoinspección semanal de las viviendas. También distribuyeron materiales educativos y de reforzamiento de los comportamientos promovidos.

Diseño y aplicación de una estrategia local de comunicación y movilización social. La estrategia de comunicación tuvo como objetivos involucrar a la población en el análisis crítico de las situaciones de riesgo relacionadas con la presencia de $A$. aegypti en la localidad, la divulgación de las actividades y la promoción de comportamientos específicos. Los GTC convocaron a los miembros de la comunidad a participar en el diseño de la estrategia de co- municación, la elaboración de mensajes, la definición de los medios y la determinación de responsabilidades para su aplicación. Se utilizó la guía para la elaboración de estrategias de comunicación popular propuesta por Vidal (18) y se fomentó la creatividad de los grupos en la elaboración de los materiales informativos.

Vigilancia comunitaria del dengue. En cada circunscripción se elaboraron mapas del riesgo de transmisión del dengue, en los que se ubicaron los sitios potenciales de cría de $A$. aegypti identificados por los líderes comunitarios, los criaderos del mosquito detectados por el personal del programa de control del vector, las familias con antecedentes de dengue, las personas con fiebre de etiología no precisada y los viajeros provenientes de zonas endémicas de dengue. Cuando se detectaba un foco de A. aegypti se intensificaban las acciones comunitarias de búsqueda de casos febriles y la elimi- 
nación de posibles criaderos en la manzana donde se detectó el foco y en las manzanas vecinas. El médico y el enfermero de la familia mantuvieron el seguimiento de las personas con fiebre hasta llegar a un diagnóstico diferencial o indicar la prueba serológica de detección de anticuerpos IgM contra el dengue a los cinco días del inicio de la fiebre. Se estableció el aislamiento domiciliario de los casos sospechados y el ingreso hospitalario en el IPK de los casos positivos. Los viajeros provenientes de áreas endémicas se registraron y siguieron durante la semana siguiente a su arribo; en caso de que refirieran fiebre eran remitidos al IPK para su ingreso y seguimiento clínico.
Evaluación participativa. En cada encuentro o taller se evaluó el desarrollo de las actividades programadas. Adicionalmente se dedicó un taller a discutir y adaptar el método de evaluación de la participación comunitaria propuesto por Rifkin y colaboradores (19). Este método se basa en la evaluación de cinco dimensiones básicas: el liderazgo, la identificación de necesidades, la organización, la gestión y la movilización de recursos. Un grupo de discusión en el que participaron tanto especialistas del IPK cómo líderes de la localidad tuvo a su cargo la adaptación de la guía de preguntas y la escala propuestas por Schmidt y Rifkin (20). En el cuadro 1 se muestran las preguntas y la escala establecida para el análisis de la participación comunitaria en la prevención del dengue durante las evaluaciones anuales realizadas.

\section{Recolección y análisis de la información}

En cada taller o encuentro se designó un relator encargado de tomar nota de los elementos fundamentales y de los acuerdos tomados. Las actividades desarrolladas, así como la participación lograda, se registraron y archivaron en un modelo diseñado a tal efecto. Para el procesamiento de la información recogida se utilizó la técnica de análisis de contenidos cualitativos (21). Mediante la codificación de los

CUADRO 1. Preguntas y escala para la autoevaluación de la participación comunitaria en la prevención del dengue durante las evaluaciones anuales realizadas

\begin{tabular}{|c|c|c|c|c|c|c|}
\hline \multirow[b]{2}{*}{ Dimensión } & \multicolumn{5}{|c|}{ Escala } & \multirow[b]{2}{*}{ Preguntas para la evaluación } \\
\hline & 1: ninguna & 2: poca & 3: media & 4: muy buena & 5: amplia & \\
\hline Liderazgo & $\begin{array}{l}\text { Liderazgo único } \\
\text { dentro del sector } \\
\text { salud }\end{array}$ & $\begin{array}{l}\text { Liderazgo } \\
\text { compartido dentro } \\
\text { del sector salud }\end{array}$ & $\begin{array}{l}\text { Liderazgo } \\
\text { compartido con } \\
\text { otros sectores }\end{array}$ & $\begin{array}{l}\text { Liderazgo } \\
\text { compartido con } \\
\text { líderes } \\
\text { comunitarios }\end{array}$ & $\begin{array}{l}\text { Liderazgo } \\
\text { compartido y } \\
\text { representativo de } \\
\text { la mayoría }\end{array}$ & $\begin{array}{l}\text { ¿Hubo coordinación única o } \\
\text { compartida? } \\
\text { ¿Quién decide el curso de las } \\
\text { acciones? } \\
\text { ¿Qué intereses están } \\
\text { representados? }\end{array}$ \\
\hline Organización & $\begin{array}{l}\text { Inexistencia de } \\
\text { grupos } \\
\text { heterogéneos }\end{array}$ & $\begin{array}{l}\text { GTC }^{a} \text { inactivo e } \\
\text { impuesto por el } \\
\text { CS }^{b}\end{array}$ & $\begin{array}{l}\text { GTC impuesto } \\
\text { por el CS pero } \\
\text { completamente } \\
\text { activo }\end{array}$ & $\begin{array}{l}\text { El GTC coopera } \\
\text { con organizacio- } \\
\text { nes comunitarias }\end{array}$ & $\begin{array}{l}\text { Las organizacio- } \\
\text { nes comunitarias } \\
\text { forman parte del } \\
\text { GTC }\end{array}$ & $\begin{array}{l}\text { ¿Quién toma las decisiones en } \\
\text { cuanto a la organización? } \\
\text { ¿Cómo está organizado el } \\
\text { trabajo dentro de la comunidad? }\end{array}$ \\
\hline $\begin{array}{l}\text { Movilización de } \\
\text { recursos }\end{array}$ & $\begin{array}{l}\text { Poca movilización } \\
\text { de recursos } \\
\text { comunitarios }\end{array}$ & $\begin{array}{l}\text { El GTC no decide } \\
\text { sobre la } \\
\text { utilización de los } \\
\text { recursos }\end{array}$ & $\begin{array}{l}\text { El GTC reúne, } \\
\text { pero no controla } \\
\text { los recursos }\end{array}$ & $\begin{array}{l}\text { El GTC controla } \\
\text { los recursos } \\
\text { reunidos por la } \\
\text { comunidad }\end{array}$ & $\begin{array}{l}\text { El GTC decide } \\
\text { sobre la } \\
\text { utilización de los } \\
\text { recursos de } \\
\text { acuerdo con las } \\
\text { necesidades } \\
\text { comunitarias }\end{array}$ & $\begin{array}{l}\text { ¿Existe una contribución de la } \\
\text { comunidad en las actividades } \\
\text { (dinero, tiempo, otros)? } \\
\text { ¿Quién decide el destino de los } \\
\text { recursos? } \\
\text { ¿El destino está en función de } \\
\text { las necesidades de la } \\
\text { comunidad? }\end{array}$ \\
\hline $\begin{array}{l}\text { Gestión y toma } \\
\text { de decisiones }\end{array}$ & $\begin{array}{l}\text { Inducida por el } \\
\text { personal de salud }\end{array}$ & $\begin{array}{l}\text { A cargo del CS } \\
\text { supervisado por } \\
\text { el personal de } \\
\text { salud }\end{array}$ & $\begin{array}{l}\text { Autogestión del } \\
\text { CS con poca } \\
\text { participación de } \\
\text { los GTC }\end{array}$ & $\begin{array}{l}\text { Autogestión del } \\
\text { CS con } \\
\text { participación } \\
\text { activa de los GTC }\end{array}$ & $\begin{array}{l}\text { Autogestión de } \\
\text { los GTC en } \\
\text { vinculación con } \\
\text { el CS }\end{array}$ & $\begin{array}{l}\text { ¿Cómo está estructurada la } \\
\text { gestión? } \\
\text { ¿A qué niveles se da la toma de } \\
\text { decisiones? }\end{array}$ \\
\hline $\begin{array}{l}\text { Identificación de } \\
\text { las necesidades }\end{array}$ & $\begin{array}{l}\text { Impuestas desde } \\
\text { afuera por el } \\
\text { personal de salud }\end{array}$ & $\begin{array}{l}\text { Domina el punto } \\
\text { de vista del CS }\end{array}$ & $\begin{array}{l}\text { El CS tiene en } \\
\text { cuenta los puntos } \\
\text { de vista de la } \\
\text { comunidad }\end{array}$ & $\begin{array}{l}\text { El GTC } \\
\text { representa } \\
\text { activamente los } \\
\text { puntos de vista } \\
\text { de la población }\end{array}$ & $\begin{array}{l}\text { Los miembros de } \\
\text { la comunidad se } \\
\text { involucran en la } \\
\text { identificación de } \\
\text { necesidades }\end{array}$ & $\begin{array}{l}\text { ¿Quiénes participaron en la } \\
\text { identificación de las } \\
\text { necesidades? } \\
\text { ¿Cómo las necesidades } \\
\text { identificadas representan los } \\
\text { puntos de vista de la población? }\end{array}$ \\
\hline
\end{tabular}

a GTC: grupo de trabajo comunitario.

${ }^{b}$ CS: consejo de salud. 
datos se determinaron los términos, las expresiones más frecuentes y los conceptos clave relacionados con la participación, la satisfacción, la toma de conciencia y la competencia de los participantes.

\section{RESULTADOS}

Los talleres de capacitación permitieron mejorar la relación entre los participantes y crear un clima de confianza entre ellos para el intercambio y el análisis de las experiencias. Se fomentó el trabajo en grupos pequeños para favorecer la colaboración de todos los participantes y se establecieron reglas para el trabajo grupal, con énfasis en el respeto a las opiniones diferentes. Las evaluaciones de los talleres fueron altamente favorables y reflejaron la satisfacción por la forma en que se trabajó y los cambios en el grado de conciencia, competencia y confianza de los participantes (cuadro 2).

El trabajo comunitario avanzó de diversas maneras, de acuerdo con las capacidades y los intereses de las comunidades de cada circunscripción. Se decidió comenzar por una circunscripción (la No. 20); las otras dos circunscripciones comenzaron el trabajo una vez que los coordinadores se sintieron preparados para aplicar lo aprendido en la práctica.

El diagnóstico participativo se realizó como parte del análisis de la situación de salud y, además de identificar los problemas, permitió reflexionar sobre sus causas y consecuencias. La información se agrupó según tres aspectos: sociales, de higienización y los relacionados con el vector. En lo social se planteó la poca motivación - especialmente de los jóvenes- y la poca divulgación de las actividades educativas y de control del vector que se planificaron; sobre la higienización se analizó la presencia de vertederos de basura, así como su asociación con deficiencias en la recogida de los desechos sólidos y el insuficiente número de colectores de basura en la zona. En cuanto al control del vector, se identificaron deficiencias en el trabajo del personal de la campaña anti-Aedes,

CUADRO 2. Frases seleccionadas expresadas por los coordinadores de los grupos de trabajo comunitario en los talleres de capacitación y evaluación

\begin{tabular}{ll}
\hline Categoría & \multicolumn{1}{c}{ Frases } \\
\hline Satisfacción & "la forma en que se ha impartido te da ganas de vivir, te da ganas de hacer, \\
& rompe la rutina" \\
Competencia & "ahora tenemos herramientas que antes no teníamos para el trabajo diario" \\
& "las técnicas que aprendimos nos ayudaron a llegar mejor a la comunidad" \\
& "me sirvió para orientarme, sensibilizar a las personas para unirnos más y para \\
& que todos sintamos responsabilidad y la compartamos" \\
Concientización & "yo creía que tenía todo muy claro y ahora veo que me faltaba integrar varios \\
& conceptos" \\
& "nos dio una visión más amplia de nuestro trabajo" \\
& "estamos despertando una parte de nosotros, a veces hacemos las cosas y no \\
& tenemos conciencia de cómo" \\
& "el solo hecho de dedicar el tiempo a discutir e intercambiar ya es importante" \\
& "me dio una nueva concepción para el trabajo, dándole mayor importancia a la \\
& participación, la intersectorialidad y al trabajo en grupo" \\
& "hay que buscar sistematicidad en las actividades y creatividad" \\
\hline
\end{tabular}

problemas de accesibilidad a los tanques de agua elevados y la deficiente hermeticidad y cuidado de los tanques de agua ubicados dentro de las viviendas. Durante la evaluación, los coordinadores plantearon criterios novedosos, como "los diagnósticos permitieron trabajar con la población por territorios y por grupos de edad", "la forma en que se trabajó dio la oportunidad de intercambiar con la gente", "el diálogo que se estableció fue una experiencia totalmente nueva" y "el trabajo en colectivo fue algo nuevo y muy positivo".

Los planes de acción abarcaron actividades de limpieza y embellecimiento de jardines, parques y otros sitios comunes, la transformación de terrenos baldíos (mediante la ubicación de puntos de ventas de frutas y vegetales, creación de terrenos deportivos y la construcción de almacenes, entre otras medidas), la realización de actividades recreativas acompañadas de mensajes de promoción de salud, y la organización de visitas domiciliarias para la detección de situaciones de riesgo ambiental y la negociación de comportamientos. Aunque durante la capacitación se promovió un enfoque holístico e integrador para lograr una comunidad saludable, solo en una de las circunscripciones (la No. 20) se logró desarrollar las acciones con este enfoque. Las otras dos circunscripciones concibieron sus planes de acción a partir de los riesgos ambientales o entomológicos identificados (cuadro 3).

La estrategia de comunicación comprendió la divulgación mediante volantes de algunos servicios subutilizados por la comunidad, como la venta de tapas para tanques de agua y los servicios de recogida de escombros y residuales sólidos. Se elaboraron materiales informativos gráficos (con dibujos y caricaturas) para la reflexión en reuniones comunitarias y para colocar en murales y viviendas. Los mensajes estuvieron encaminados a la eliminación de los recipientes inútiles de las viviendas y sus alrededores, el tapado, reparación, limpieza y cepillado de los tanques de agua, la limpieza de las áreas comunes y la realización de la autoinspección semanal de las viviendas. Se aprovecharon sitios públicos y reuniones de la comunidad para el intercambio de información.

Como medida de vigilancia comunitaria se elaboraron mapas de riesgo 
CUADRO 3. Conceptos de trabajo del consejo de salud y de los grupos de trabajo comunitario (GTC) en las tres circunscripciones de estudio

\begin{tabular}{|c|c|}
\hline Nivel & Conceptos de trabajo \\
\hline Consejo de salud del área 26 de Julio & $\begin{array}{l}\text { Enfoque intersectorial. Cada sector compartió } \\
\text { información sobre la situación entomológica, casos } \\
\text { importados sospechados de dengue, riesgos ambientales y } \\
\text { otras necesidades identificadas por los GTC. El consejo de } \\
\text { salud elaboró, ejecutó y monitoreó el plan de acción } \\
\text { intersectorial, que comprendió el apoyo a las actividades } \\
\text { planificadas por los GTC. }\end{array}$ \\
\hline
\end{tabular}

GTC de la circunscripción No. 20

Enfoque de comunidad saludable. La prevención del dengue se vinculó a las acciones del programa de promoción de salud, que comprendió, entre otras, la limpieza y ornamentación de la comunidad y acciones para elevar la calidad de vida de los adultos mayores.

GTC de la circunscripción No. 51

Enfoque de riesgo ambiental. Para el control de Aedes aegypti se emprendieron acciones encaminadas principalmente a la eliminación de posibles criaderos del vector y se incorporaron al programa de saneamiento ambiental.

GTC de la circunscripción No. 40

Enfoque de riesgo entomológico. Las acciones se realizaron en un perímetro de 100 metros alrededor de las viviendas en que se detectaron focos de $A$. aegypti. que fueron ubicados en los consultorios del médico y el enfermero de la familia, actualizados por los enfermeros o los líderes de los GTC. Mensualmente se procesó y analizó la información y se envió un informe al vicedirector de higiene y epidemiología del área de salud, por ser quien representaba a este sector en el consejo de salud.

Las evaluaciones realizadas permitieron constatar cambios en el concepto de participación de los involucrados en el proceso y en las cinco dimensiones de la participación evaluadas. Al inicio del período, los miembros de la comunidad consideraban la participación como "la colaboración y el apoyo a todas las acciones programadas" o "la intervención de forma consciente en una actividad o trabajo de grupo en objetivos bien definidos para darle solución a un problema determinado". En la evaluación realizada dos años después de iniciada la intervención, el concepto de participación construido colectivamente por los facilitadores era de un proceso "que implica trabajar con la heterogeneidad de la población, tener en cuenta lo que opinan de sus problemas y de las soluciones que pueden tener", "de trabajo colectivo que requiere de motivación y de personas que lideren ese proceso", "donde es necesario un diálogo constante entre todos los involucrados" y "que crea un compromiso con el resto de las personas que esperan acciones y cambios en nosotros".

En la figura 2 se muestran los resultados de la evolución de los cambios ocurridos en la participación comunitaria durante la aplicación de la estrategia. El liderazgo del personal de salud pasó de ser paternalista a compartirse con los líderes de la comunidad, ya que se tuvieron en cuenta sus opiniones en el planeamiento de las acciones. La organización basada fundamentalmente en las estructuras y actividades de APS se hizo más participativa con la constitución de los GTC, que funcionaron en coordinación con el consejo de salud. Se involucró a la población en la identificación de las necesidades mediante los diagnósticos participativos. La gestión, que era inducida por el personal de salud, pasó a formas más participativas y se crearon mecanismos para la toma de decisiones colectivas en los GTC. La movili- zación de recursos, inicialmente limitada al sector salud, se amplió a otros sectores $y$, posteriormente, al aporte voluntario de los miembros de la comunidad.

Estos resultados contribuyeron a que al final de dos años de intervención, el índice de depósitos con larvas o pupas de $A$. aegypti por 100 viviendas inspeccionadas se redujera en $79 \%$ y no se detectaron casos de dengue en ninguna de las tres circunscripciones.

\section{DISCUSIÓN}

El concepto de educación popular adoptado facilita el empoderamiento comunitario y su metodología se basa en investigaciones sobre la acción participativa $(17,21,22)$. Mediante un proceso de reflexión crítica, de análisis de situaciones cotidianas y del comportamiento de la vida en sus comunidades, las personas se constituyen en sujetos de su propio desarrollo. La participación se logra mediante el aprendizaje, el estudio y la investigación de los problemas, la búsqueda de las causas, la formulación de propuestas y la ejecución y evaluación del proceso vivido. Es así cómo las personas de una localidad pasan de ser simples informantes o participantes en la ejecución de acciones a constituirse en actores fundamentales en la toma de decisiones (22).

Uno de los elementos más altamente valorados en la práctica de la educación popular está ligado a su proceso motivador, inventivo y provocativo, que prepara a los miembros de la comunidad para los cambios futuros. Los materiales, juegos y técnicas participativas empleados en los talleres favorecieron la interacción y elevaron la conciencia de los participantes sobre los problemas locales. Además, les permitió valorar mejor los recursos no materiales, tales como la unión de los miembros del grupo, el ambiente de alegría, el reconocimiento mutuo, el cumplimiento de los compromisos asumidos, la afectividad y la receptividad de criterios diferentes y el hecho de sentir que se toman en cuenta las opiniones personales. Otro punto im- 
FIGURA 2. Cambios en la participación comunitaria en la prevención del dengue y el control de Aedes aegypti

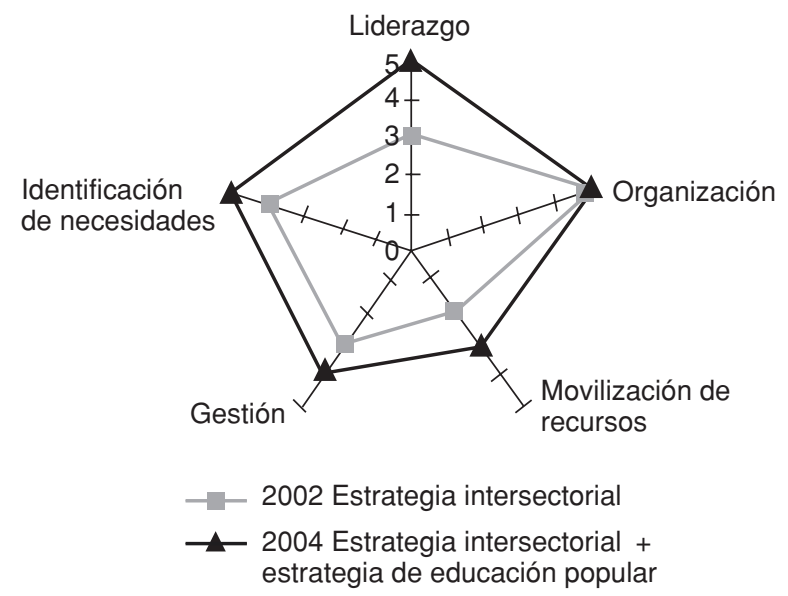

portante es que al establecerse espacios de encuentro, se elevó el sentido de comunidad en los pobladores.

En esta experiencia, los GTC no solo fueron protagonistas en la definición de las acciones realizadas en las comunidades, también contribuyeron en la definición de los objetivos y en la recopilación de la información necesaria para la investigación desarrollada, mientras el personal externo se concentró en tareas de asesoramiento y capacitación. El empoderamiento de los grupos para realizar los diagnósticos participativos programados facilitó que los miembros de la comunidad se involucraran en la toma de decisiones y permitió adquirir las destrezas necesarias para la elaboración participativa de los planes de acción y de la estrategia de comunicación. Sin embargo, es importante tener en cuenta que el empoderamiento comunitario trae consigo algunas dificultades, ya que de cierta forma implica que simultáneamente se reduzca el poder y la autonomía de una parte de los miembros de la comunidad (23). Algunos gobiernos tienen la voluntad de apoyar la participación comunitaria, pero no al costo de perder su autoridad. Es por esto que los ideales de la participación de la población para promover la salud y el desarrollo comunitarios se pueden aplicar más fácilmente en sociedades democráticas (24). El proceso de em- poderamiento promovido mediante la educación popular entraña la revisión de visiones, percepciones, actitudes y relaciones sociales a partir de una ética que promueve valores de justicia, equidad y solidaridad humana (25).

En este proceso las comunidades modifican los mecanismos movilizadores y orientadores - utilizados habitualmente para lograr la participación comunitaria- por la elaboración comprometida, respetuosa de la diversidad y la autonomía, y generadora de propuestas, decisiones y mecanismos sociales que promueven la participación (26). El desarrollo de programas comunitarios basados en este enfoque requiere de cambios en las concepciones predominantes y la capacitación del personal de salud, de los líderes comunitarios y de los funcionarios del gobierno local. Se hace necesario preparar al personal de salud para que comparta el poder - que históricamente se les ha otorgado- con los miembros de la comunidad, para que estos asuman y compartan responsabilidades en el cuidado y la promoción de la salud. Los gobiernos locales deben dar un mayor espacio a la participación comunitaria.

La importancia de la evaluación participativa en este proceso, así como su valor educativo, están bien documentados (27). La metodología aplicada en este trabajo para evaluar la participa- ción comunitaria se ha utilizado con anterioridad en investigaciones sobre APS (20) y, con algunas modificaciones, en la evaluación de las capacidades comunitarias para la promoción de salud (28). Como metodología participativa, los participantes toman conciencia de las dificultades a medida que se aplica y se introducen cambios inmediatos en la planificación de la etapa siguiente. Sin embargo, se debe tener en cuenta que el tiempo que transcurre entre el inicio de un proceso de educación popular, el logro de la participación comunitaria activa en la toma de decisiones y los cambios en la salud puede ser considerable. Se ha planteado que con el desarrollo comunitario, en cinco años se pueden ver cambios en el comportamiento específico promovido mediante campañas intensivas de comunicación (29). Esto quiere decir que se requiere de un tiempo mayor que el contemplado en este estudio para poder hacer una valoración final de la intervención realizada.

Los resultados alcanzados en esta experiencia demuestran que la estrategia empleada, basada en la educación popular, la adecuada comunicación y el trabajo sistemático de grupo, organizado y creativo, permitió reducir el nivel de infestación con el mosquito vector mediante el aumento de la participación de la comunidad en la toma de decisiones, el fortalecimiento de las competencias de los equipos médicos y de los GTC para liderar procesos participativos en sus comunidades y la elevación de la confianza de los miembros de la comunidad en sus posibilidades de llevar a cabo transformaciones con recursos propios y apoyo intersectorial.

Para lograr una mayor eficacia de las acciones de control del dengue, los programas e intervenciones deben prever el empoderamiento efectivo de la comunidad para lograr una mayor participación comunitaria e intersectorial en la toma de decisiones. Los programas de formación y capacitación del personal de salud deben incluir aspectos conceptuales y metodológicos relacionados con la educación popular y fomentar habilidades para la conducción de procesos participativos. 


\section{REFERENCIAS}

1. Kourí G. El dengue, un problema creciente de salud en las Américas. Rev Panam Salud Publica. 2006;19:143-5.

2. Guzmán MG, Kourí G. Dengue: an update. Lancet Infect Dis. 2002;2:33-42.

3. Gubler DJ, Clark GG. Community involvement in the control of Aedes aegypti. Acta Trop. 1996;61:169-79.

4. Gubler DJ, Clark GG. Community-based integrated control of Aedes aegypti: a brief overview of current programs. Am J Trop Med Hyg. 1994;50:50-60.

5. Renganathan E, Parks P, Lloyd L, Nathan MB, Hosein E, Odugleh A, et al. Towards sustaining behavioural impact in Dengue prevention and control. Dengue Bull. 2003;27:6-12.

6. Parks W, Lloyd L. Planificación de la movilización y comunicación social para la prevención del dengue. Guía paso a paso. Washington, DC.: OPS; 2004.

7. Organización Panamericana de la Salud. Nueva generación de programas de prevención y control del dengue en las Américas. Washington, D.C.: OPS; 2002. (Documento OPS/HCP/206/02).

8. Parks W, Lloyd L, Nathan M, Hosein E, Odugleh A, Clark GG, et al. International experiences in social mobilization and communication for Dengue prevention and control. Dengue Bull. 2004;28:1-8.

9. Freire P. La educación como práctica de la libertad. México: Siglo XXI; 1976.

10. Duarte M, Freese E. Discursos e práticas referentes ao processo de participação comunitária nas ações de educação em saúde: as ações de mobilização comunitária do PCDEN/PE. Cad Saude Publica. 1998;14: 59-68.

11. Mourão E. Educação popular como instrumento de reorientação das estratégias de con- trole das doenças infecciosas e parasitárias. Cad Saude Publica. 1998;14:39-57.

12. Pereira $A$. As tendências pedagógicas e a práctica educativa nas ciencias de saúde. Cad Saude Publica. 2003;19:1527-34.

13. Guzmán MG, Peláez O, Kourí G, Quintana I, Vázquez S, Pentón M, et al. Caracterización final y lecciones de la epidemia de dengue 3 en Cuba, 2001-2002. Rev Panam Salud Publica. 2006;19:282-9.

14. Valdés L, Guzmán MG, Kourí G, Delgado J, Carbonell I, Cabrera MV, et al. Epidemiology of dengue and hemorrhagic dengue in Santiago, Cuba, 1997. Rev Panam Salud Publica. 1999;6:16-25.

15. Peláez O, Guzmán MG, Kourí G, Pérez R, San Martín JL, Vázquez S, et al. Dengue 3 epidemic, Havana, 2001. Emerg Infect Dis. 2004;10:719-22.

16. Sánchez L, Pérez D, Pérez T, Sosa T, Cruz G, Kourí $\mathrm{G}$, et al. Intersectoral coordination in Dengue prevention. A pilot project in Playa municipality, Havana City, Cuba. Int J Trop Med. 2005;10:82-91.

17. Shrestha S. A conceptual model for empowerment of the female community health volunteers in Nepal. Educ Health. 2003;16: 318-27.

18. Vidal JR. Guía para la elaboración de una estrategia de comunicación popular. En: Comunicación y educación popular: selección de lecturas. La Habana: Caminos; 2004. Pp. 21-9.

19. Rifkin S, Muller F, Bichman W. Primary health care: on measuring participation. Soc Sci Med. 1988;26:931-40.

20. Schmidt DH, Rifkin SB. Measuring participation: its use as a managerial tool for district health planners based on a case study in Tanzania. Int J Health Plan Manag. 1996;11: 345-58.

21. Gómez M. Análisis de contenido cualitativo y cuantitativo: definición, clasificación y metodología. Rev Ciencias Humanas. 1999, 20. Hallado en: http://www.utp.edu.co/ $\sim$ chumanas/revistas/revistas/rev20/gomez. $\mathrm{htm}$. Acceso el 19 de noviembre de 2003.

22. Baum F, MacDougall C, Smith D. Participatory action research. J Epidemiol Community Health. 2006;60:854-7.

23. Drury J, Cocking C, Beale J, Hanson C, Raplay F. The phenomenology of empowerment in collective action. Br J Soc Psychol. 2005;44:309-28.

24. Espino F, Koops V, Manderson L. Community participation and tropical disease control in resource-poor settings. Geneva: WHO; 2004

25. Rebellato JL. La participación como territorio de contradicciones éticas. En: Ética de la autonomía. Montevideo: Rosa Vein; 1997.

26. D'Angelo O. Relaciones entre política, la subjetividad social y una ética emancipatoria para el desarrollo humano. En: Hernández $\mathrm{CN}$, ed. Trabajo comunitario: selección de lecturas. La Habana: Caminos; 2005. Pp. 41-50.

27. Kroeger A, Franken HP. The educational value of participatory evaluation of primary health care programs: an experience with four indigenous populations in Ecuador. Soc Sci Med. 1981;15B:535-9.

28. Gibbon M, Labonte R, Laverack G. Evaluating community capacity. Health Soc Care Community. 2002;10:485-91.

29. Leeuw E. Concepts in health promotion: the notion of relativism. Soc Sci Med. 1989;29: $1281-8$.

Manuscrito recibido el 18 de mayo de 2007. Aceptado para publicación, tras revisión, el 5 de noviembre de 2007.
ABSTRACT

\section{A community education strategy to promote participation in dengue prevention in Cuba}

Objectives. To document the process and analyze the results of implementing a strategy aimed at increasing community participation in the fight against the dengue mosquito vector.

Methods. From May 2002 to May 2004, an intervention was implemented to advance social action against dengue in three districts of the municipality of Playa, La Habana, Cuba. A learning group and community working groups (CWG) were organized in each location. A community education model was followed that combines putting the plan of action into practice, with sessions to reflect and learn from the results, and then reworking of the actions for subsequent stages. Diagnostic tools were developed for communities, preventative actions, communication, surveillance, and evaluation. Changes in participation were identified by applying the content analysis technique to the documents and through interviews with key informants.

Results. The community work advanced at a pace relative to the abilities and interests of each community with different areas of focus: healthy community, environmental risk, and entomological risk. Positive changes in the concept of participation were obtained, according to the five areas evaluated: leadership, needs assessment, organization, management, and mobilization of resources. At the end of two years of intervention, the rate of Aedes aegypti larvae and pupae deposits found per 100 households had declined $79 \%$ and cases of dengue were not detected in any of the districts. Conclusions. This strategy reduced mosquito vector infestation levels by increasing community participation in decision-making and strengthening the competencies of the medical teams and CWGs so that they lead participative processes in the community and raised confidence in their ability to achieve change with the appropriate resources and intersectoral support.

Key words Population education, consumer participation, dengue, vector control, Aedes, Cuba. 